\title{
The thermodynamics of the FRW universe in scalar-twist gravitational theories
}

\author{
T Azizi
}

Department of Physics, Faculty of Basic Sciences, University of Mazandaran, Babolsar, Iran

E-mail: t.azizi@umz.ac.ir

\begin{abstract}
In this paper, we study the validity of the laws of thermodynamics in the form of a far parallel gravitational theory with an incomplete coupling between curves and scalar fields. To this end, we consider the FRW flat world, showing that the first and second laws of thermodynamics lie in its dynamic apparent horizon. We further assume that the universe is enclosed by the cosmological event horizon, such that in this case the first law of thermodynamics is valid, but the second law of thermodynamics is applied to the selected incomplete model, depending on the energy-momentum tensor components derived.
\end{abstract}

Keywords: cosmology, modified gravity, far parallel gravity, incomplete coupling, thermodynamics

For full article, refer to the Persian section 